\title{
Pheochromocytoma as a rare hypertensive complication rarely associated with pregnancy: Diagnostic difficulties (Review)
}

\author{
IRINA PACU ${ }^{1,2}$, NIKOLAOS ZYGOUROPOULOS ${ }^{2}$, CRISTIAN GEORGE FURAU ${ }^{3,4}$, \\ DAN NAVOLAN $^{5,6}$, DELIA MIRELA TIT ${ }^{7}$, CRINGU A. IONESCU $^{1,2}$, \\ ANCA PANTEA STOIAN ${ }^{1,8}$, AIDA PETCA ${ }^{1,9}$ and MIHAI DIMITRIU ${ }^{1,2}$ \\ ${ }^{1}$ Department of Obstetrics and Gynecology, 'Carol Davila' University of Medicine and Pharmacy, 050474 Bucharest; \\ ${ }^{2}$ Department of Obstetrics and Gynecology, 'Sf. Pantelimon’ Emergency Clinical Hospital, 021623 Bucharest; \\ ${ }^{3}$ Department of Obstetrics and Gynecology, Arad County Clinical Emergency Hospital, 310037 Arad; \\ ${ }^{4}$ Department of Obstetrics and Gynecology, 'Vasile Goldis' Western University of Arad, 310025 Arad; \\ ${ }^{5}$ Department of Obstetrics and Gynecology, 'Victor Babeș' University of Medicine and Pharmacy, 300041 Timișoara; \\ ${ }^{6}$ Department of Obstetrics and Gynecology, Timisoara Municipal Clinical Emergency Hospital, 300254 Timișoara; \\ ${ }^{7}$ Department of Pharmacy, University of Oradea, 410087 Oradea; ${ }^{8}$ Diabetes, Nutrition and Metabolic Diseases, \\ 'N. C. Paulescu' National Institute for Diabetes, Nutrition and Metabolic Diseases, 020475 Bucharest; \\ ${ }^{9}$ Department of Obstetrics and Gynecology, 'Elias' Emergency University Hospital, 011461 Bucharest, Romania
}

Received July 19, 2021; Accepted August 18, 2021

DOI: $10.3892 /$ etm.2021.10780

\begin{abstract}
This review provides a brief clinically relevant review of pheochromocytoma in pregnancy, to raise awareness among doctors in obstetrics and the aim is to serve as the first point of reference when confronted by their presence. Pheochromocytomas are neuroendocrine, catecholamine-secreting tumours. Despite having the highest incidence rate among other hormone-secreting adrenal tumours, they remain rare especially when associated with pregnancy. The non-specific presentation of pheochromocytomas, the difficulties in their diagnosis during pregnancy as well as the high maternal and fetal mortality rates associated with them, present a challenge. Clinical suspicion and meticulous patient history-taking remain the primary lines of defense, while biochemical proof of catecholamine excess (or their metabolites) and imaging-based localisation of the tumour are required for diagnosis. Antenatal diagnosis and complete localisation of the tumour increase the likelihood
\end{abstract}

Correspondence to: Dr Mihai Dimitriu, Department of Obstetrics and Gynecology, 'Sf. Pantelimon' Emergency Clinical Hospital, 340-342 Pantelimon Boulevard, 021623 Bucharest, Romania

E-mail: drmihaidimitriu@yahoo.com

Abbreviations: CT, computed tomography; DOPA, dihydroxyphenylalanine; MEN2, multiple endocrine neoplasia type 2; MIBG, metaiodobenzylguanidine; MRI, magnetic resonance imaging; NF1, neurofibromatosis type I; VHL, von Hippel-Lindau

Key words: pheochromocytoma, pregnancy, catecholamine excess, secondary hypertension, multiple endocrine neoplasia type 2, neurofibromatosis type 1 of successful outcomes for both mother and newborn. Magnetic resonance imaging (MRI) remains the method of choice during pregnancy without excluding the use of ultrasound. Treatment goals should include the avoidance of hypertensive crises while maintaining adequate uteroplacental circulation. The target blood pressure is not strictly defined but is in line with the general guideline addressing chronic hypertension during pregnancy. Antihypertensive medications remain the cornerstone in managing pheochromocytoma. As a first-line, the $\alpha$-adrenergic, nonselective antagonist phenoxybenzamine is the most frequently used agent, while $\alpha 1$-selective adrenergic antagonists with or without the addition of $\beta$ - or $\beta 1$-blockers are also prescribed in certain cases, rendering calcium channel blockers as 'second-choice'. Blood-pressure control of the mother and the well-being of the fetus are determining factors in deciding the time of delivery, which is preferably conducted by Caesarean section. Excision of the tumour(s) remains the final treatment goal. Lifelong biochemical testing is required with or without medical treatment, to address mineralocorticoid or glucocorticoid deficits. Despite ever-improving positive outcome rates, pheochromocytoma associated with pregnancy remains a pathology with high mortality and morbidity rates.

\section{Contents}

1. Introduction

2. Diagnosis

3. Clinical presentation

4. Genetic aspects

5. Biochemical testing

6. Localisation and biopsy 
7. Management

8. Prognosis and follow-up

9. Conclusions

\section{Introduction}

Pheochromocytomas are neuroendocrine tumours arising from chromaffin cells derived from the neural crest. Consequently, they are mostly catecholamine-secreting tumours, highly vascular, and usually located in the adrenal medulla (1). Tumours of extra-adrenal origin are often referred to as paragangliomas or extra-adrenal pheochromocytomas (2). In this review, we will refer to both as pheochromocytoma for the sake of brevity and specify when necessary.

In authoring this review, we initially conducted a search in Pubmed, UpToDate and ResearchGate using as keywords 'pheochromocytoma', 'neuroendocrine tumors', 'paraganglioma', 'neurofibromatosis type I' and 'pregnancy' in several combinations of them with a single constant: 'pregnancy'. The time frame selected was between January 2000 and September 2020 considering that older studies were likely to have been included in the most recent ones. Studies that did not concern pheochromocytoma during pregnancy as well as studies that were included or referred to by more recent studies were excluded. A conscious attempt to provide clinically useful information was made-as much as possible-instead of focusing on details that may confuse the reader especially in view of the rarity of this pathology encountered in obstetrics.

Despite pheochromocytomas having the highest incidence rate among other hormone-secreting adrenal tumours, they remain rare, especially when associated with pregnancy, with a reported incidence rate of 0.32 per 100,000 pregnant women (3). Approximately 30-40\% are part of a hereditary syndrome (germline mutation) (4), 40-50\% are related to identifiable somatic mutations (in one or more of the 20 susceptibility genes identified to date), leaving a quarter of them considered as sporadic tumours (5).

\section{Diagnosis}

Pheochromocytoma is diagnosed by biochemical tests followed by anatomical localisation of the secreting tumour(s) utilising imaging techniques (6). Although pregnancy does not impact biochemical testing, it renders magnetic resonance imaging (MRI) and/or ultrasound scan as the preferred imaging techniques (7) for the anatomical localisation of the tumour precluding instead computer tomography, ${ }^{123}$ I-metaiodobenzylguanidine (MIBG) scintigraphy, or the use of ionising radiation with alternative contrast media (6).

Investigations should be undertaken based on clinical suspicion or in cases of incidentally discovered adrenal masses. The absence of symptoms does not exclude the possibility of a pheochromocytoma that does not secrete catecholamines. Except in the cases where adrenal masses are incidentally identified, certain patient characteristics as well as presenting signs and symptoms should alert clinicians in pursuing the exclusion or confirmation of pheochromocytoma.

\section{Clinical presentation}

Signs and symptoms are diverse, non-specific (6) and common between the pregnant and non-pregnant population, while diagnosis can be challenging due to the low prevalence and overlapping features with other common disturbances in pregnancy (7), such as hyperemesis, gestational diabetes, threatened miscarriage or pre-eclampsia. Symptom intensity can range from minor to severe/life-threatening and from paroxysmal to persistent (8), becoming more pronounced with increasing gestational age.

Hypertension is the most prominent feature of pheochromocytoma (2), present in approximately $95 \%$ of patients and related to catecholamine excess and the main symptom during pregnancy. Hypertension can be persistent $(\sim 29 \%)$ or episodic ( $48 \%)$ (9). In episodic hypertension, periods of hypotension can be identified in as many as $50 \%$ of the patients (10) Nevertheless, the absence of hypertension does not rule out pheochromocytoma since $\sim 13 \%$ of patients are normotensive (6).

Specific signs that should singularly raise sufficient suspicion to dictate pheochromocytoma investigations include orthostatic hypotension in an otherwise hypertensive pregnant woman (8) or presence of resistant hypertension at a young age ( $<20$ years of age), especially in the absence of other related factors. Similarly, presentation of hypertension $<20$ weeks of gestation, paroxysmal hypertension, and/or adrenergic spells (episodic palpitations, diaphoresis, pallor, tremor) are more strongly associated with the presence of a pheochromocytoma rather than gestational hypertension or preeclampsia. Conversely, ankle edema, proteinuria, increased serum uric acid, signs of hemolysis, elevated liver enzymes, and low platelet counts, especially in the context of advanced pregnancy, are signs more commonly associated with gestational hypertension or preeclampsia (8).

Paroxysmal headaches and palpitations attributed to adrenergic spells are variably the second and third most common symptoms in cases of pheochromocytoma, especially during pregnancy. Other non-specific but common symptoms include weakness and chronic fatigue while gastrointestinal symptoms, such as nausea, constipation, vomiting, and epigastric pain, are less common (6).

Table I summarises the findings that would alter pre-test probability for pheochromocytoma during pregnancy.

\section{Genetic aspects}

From diagnosed pheochromocytomas, it is known that approximately $30-40 \%$ are hereditary and another $40-50 \%$ arise from identifiable somatic mutations in many of the 20 currently characterised susceptibility genes (5). Awareness of specific features associated with hereditary pheochromocytoma syndromes, particularly multiple endocrine neoplasia type 2 (MEN2), multiple endocrine neoplasia type 2; neurofibromatosis type I (NF1), von Hippel-Lindau (VHL), and familial paraganglioma syndromes, is crucial in enabling timely consideration, genetic counseling, and analysis. This is of particular importance in pregnant women where due to their relatively young age, the probability of a pathogenic germline mutation (30-40\%) and thus the risk of presenting a multifocal, 
Table I. Instances that merit the investigation for and exclusion of pheochromocytoma.

Signs and symptoms, from patient histories that alter the pre-test probability for pheochromocytoma during pregnancy Pre-test probability

Associated signs and symptoms

Increase

Unchanged

Decrease
Associated features of hereditary syndromes of pheochromocytoma (MEN-2A, VHL, NF1, PGL 1-5, Carney triad) in conjunction with personal and family history

Family history of pheochromocytoma

History of abnormal blood pressure response during surgical interventions

Orthostatic hypotension in a hypertensive pregnant woman

Presentation of hypertension at $<20$ weeks of gestation

Resistant hypertension at a young age ( $<20$ years)

Paroxysmal hypertension and/or classic adrenergic spells (episodic palpitations,

diaphoresis, pallor, tremor)

Paroxysmal headaches

Nausea

Proteinuria

Edema

Increased plasma uric acid level

HELLP syndrome

MEN2, multiple endocrine neoplasia type 2; NF1, neurofibromatosis type I; VHL, von Hippel-Lindau; HELLP, hemolysis elevated liver enzymes and low platelets.

recurrent, and metastatic (malignant) disease is higher (8). Knowledge of the underlying germline/somatic mutations and their clinical presentation can guide biochemical testing, specific imaging modalities, and appropriate personalised treatments. Importantly, hereditary mutations may affect the unborn child and this aspect is required to be discussed with the expecting mother.

\section{Biochemical testing}

Central to establishing the diagnosis of pheochromocytoma using biochemical investigations are confirming the presence of catecholamines (epinephrine, norepinephrine, and dopamine) or their O-methylated metabolites (metanephrine, normetanephrine, and 3-methoxytyramine respectively) in excess (7) in plasma or urine (1). The selection of a specific biochemical test is generally debated, with doubt around which molecule should be measured (catecholamines or metabolites) and in which bodily fluid (plasma or urine).

As described elsewhere (11), plasma levels of normetanephrine $<112 \mathrm{ng} /$ liter $(0.61 \mathrm{nmol} / \mathrm{l})$ and of metanephrine $<61 \mathrm{ng} / \mathrm{l}(0.31 \mathrm{nmol} / \mathrm{l})$ virtually exclude pheochromocytoma. With plasma concentrations of normetanephrine $>400 \mathrm{ng} / 1$ ( $2.19 \mathrm{nmol} / \mathrm{l})$ or of metanephrine $>236 \mathrm{ng} / \mathrm{l}(1.20 \mathrm{nmol} / \mathrm{l})$, the probability of pheochromocytoma is so high that the next step is to locate the tumour. This however does not subtract from the diagnostic value of epinephrine and norepinephrine, which at clearly elevated values ( $>2$ times the upper limit of the normal range) are also considered diagnostic (12). The superiority of metabolites over catecholamines in terms of diagnostic power may be attributed to the constant production of O-methylated metabolites from catecholamine-leaking vesicles in tumour cells, their relatively long half-lives, and lack of sympathoadrenal excitation influence.

A proposed solution to improve distinction amongst true-positive and false-positive results is the use of ratios between normetanephrine to norepinephrine or metanephrine to epinephrine especially in pregnancy during which a clonidine-suppression test is contraindicated. The higher the ratio between normetanephrine to norepinephrine $(>0.52)$ or metanephrine to epinephrine ( $>4.2)$, the higher the possibility (up to $30 \%$ ) of a true-positive result suggestive of pheochromocytoma (13). However, the reverse pattern to exclude pheochromocytoma is unreliable because some tumours secrete relatively large amounts of catecholamines compared with metanephrines (13).

To further avoid false-positive results, some authors suggest that the initial test of choice should be based on clinical suspicion and pre-test probability (a priori risk and suspicion). Accordingly, sensitive plasma metanephrine measurements $(97.9 \%$ ) with a nearly maximal negative predictive value should thus be reserved for high a priori risk (including suspected familial endocrine syndromes) (12), with attention drawn to some rare exceptions of non-functional or small tumours. However, this is not the case for the pregnant patient presenting symptoms (8) in which high specificity (true negative rate) would be more of interest.

Overall, since approximately $80 \%$ of pheochromocytoma patients present increased plasma normetanephrines and metanephrines, a negative test in low a priori risk patients would exclude the diagnosis while a 4-fold increase in high a priori risk patients (with genetic predisposition) would confirm it (13). 


\section{Localisation and biopsy}

In general, anatomic localisation of pheochromocytomas should be undertaken after biochemical confirmation. However, in recent years tumours are increasingly being identified incidentally through various imaging modalities, which prompts their biochemical or other investigation as a second step (6).

During the localisation stage, in pregnant patients, imaging investigations are usually limited to anatomic non-ionising methods such as MRI and ultrasound (7). Although ultrasonography is widely used in pregnant patients, it is associated with overall poor sensitivity due to the increasing fundal size of the uterus which can interfere with its use $(8,14)$, especially during the third trimester, potentially leading to small tumours being missed (14). However, its use may still be considered in pregnant patients presenting with unexplained hypertension in the first trimester (14).

To date, MRI is the preferred imaging modality to exclude or detect pheochromocytoma in pregnant women due to its safety (15) and similar sensitivity to computed tomography (CT) despite its limited specificity (70-80\% compared to CT) (7). Additionally, modality-specific imaging criteria can be used to suggest whether an adrenal mass is benign or not (16).

The European guideline for adrenal incidentalomas (16) claims that biopsy of an adrenal tumour should only be performed if: a) there is another extra-adrenal malignancy in the patient history; b) if the tumour is non-functioning; c) if the tumour has not been judged as benign on imaging; d) if a biopsy would change patient management.

Otherwise, surgical resection should proceed without a prior biopsy. Consequently, all things considered, in a pregnant patient biopsy would be contraindicated in the case of a functioning tumour (presenting symptoms or positive biochemical testing) because of the risk of eliciting a hypertensive crisis. The debate as to whether to perform a biopsy or not is limited to non-functioning tumours (hence incidentally identified during pregnancy) which are not judged as benign on imaging with or without a history of extra-adrenal malignancy.

Differential diagnosis. Pathologies other than preeclampsia that may present similar features to catecholamine-secreting pheochromocytomas and need to be considered in the differential diagnosis during pregnancy include carcinoid syndrome, mast cell diseases, hypoglycemia, labile essential hypertension, withdrawal from adrenergic drugs, panic attacks, paroxysmal cardiac arrhythmias (17).

\section{Management}

Once the diagnosis of pheochromocytoma has been established, definitive treatment consists of complete surgical resection of the tumour, in both malignant and non-malignant tumours (6), with the preferred method of removal being a transperitoneal laparoscopic approach especially when the tumour size is $<7 \mathrm{~cm}$ (14). However, this is not always possible during pregnancy and in some cases also in non-pregnant patients. Currently, the consensus is that when the diagnosis is made before 23 weeks of gestation, excision should be performed (7). More details regarding the surgical intervention for resection of pheochromocytoma during pregnancy have been published elsewhere (18) and will not be discussed further in this review.

Beyond 23 weeks' gestation, the dimensions of the uterus may preclude surgery, especially laparoscopy (18). Under these circumstances, medical treatment, such as adrenergic blockade, is the only available option while awaiting fetal maturation and delivery during the third trimester. Excision can either follow straight after delivery or be delayed by 2-6 weeks postpartum (7). In any case, preoperative medical management is imperative to minimise the risks related to hypertensive adrenergic crises during pregnancy as well as perioperative complications, thus improving patient outcome (19). It is important to note that the available data regarding pregnant women from which the above recommendations are drawn are small retrospective studies with an inherent risk of selection bias; especially in the case of negative outcomes (fetal demise or maternal death), it is difficult to establish whether these are attributed to the surgery or the pheochromocytoma per se. Postoperative follow-up is required at 2-6 weeks using biochemical measurements of plasma or urine metanephrines.

Timeliness and mode of delivery. The success of medical management (discussed below) and the well-being of the fetus are determining factors in deciding the time of delivery (7). Inability to control the mother's blood pressure, a strong suspicion of malignant pheochromocytoma, intrauterine growth retardation, decreased fetal movements, and decelerations on fetal cardiac rhythm are some indications that could prompt early delivery (7). Despite the lack of specific studies examining the best route of delivery, the consensus suggests that a planned Cesarean section is the best option (20).

Preoperative and medical management. The aims of medical management preoperatively or until the time of delivery are to control blood pressure and heart rate, prevent catecholamine crises due to excess release during intraoperative manipulation, and address volume contraction resulting from chronic vasoconstriction secondary to chronic catecholamine excess.

The target blood pressure quoted for a non-pregnant population is less than $130 / 80 \mathrm{mmHg}$ when seated and when standing systolic pressure greater than $90 \mathrm{mmHg}(6,15)$. In a pregnant population, recommendations are similar to those for chronic hypertension in pregnancy, i.e., $<150 / 100 \mathrm{mmHg}$ with diastolic blood pressure $>80 \mathrm{mmHg}$ in absence of end-organ damage signs or $<140 / 90 \mathrm{mmHg}$ in the case of end-organ damage signs (21).

As a first-line treatment, the $\alpha$-adrenergic, nonselective antagonist phenoxybenzamine is the most frequently used agent in pregnancy despite crossing the placenta and consequently is associated with neonatal hypotension and respiratory depression (22). Alternatively, $\alpha 1$-selective adrenergic antagonists such as doxazosin, prazosin, or terazosin are used to avoid adverse effects usually associated with unselective $\alpha$-blockade. Furthermore, in the case of doxazosin despite it also crossing the placenta no adverse effects have been reported to date (23). It is important to note that despite the wide use of $\alpha$-adrenergic blockage in pregnant women the authors were unable to find any randomized control clinical trials.

In certain circumstances such as the appearance of side effects due to $\alpha$-blockade and/or failure to control symptoms, 
the addition of a $\beta$-blocker should be considered. The preferred agent is propranolol, an unselective $\beta$-blocker. Selective $\beta 1$-blockers can be used in place of unselective $\beta$-blockers in patients with bronchospastic disease (24).

Calcium channel blockers are used as an alternative or complement to $\alpha$ - and $\beta$-blockers in patients unable to tolerate or achieve desired blood pressure control (25). Despite little experience in their use in pheochromocytoma, there is growing evidence to suggest they can potentially be used as first-line agents instead of $\alpha$ - and $\beta$-blockers, with lower morbidity and mortality (26).

Lastly, metyrosine (a tyrosine hydroxylase inhibitor), which blocks the rate-determining step metabolising tyrosine to dihydroxyphenylalanine (DOPA) in the synthesis of catecholamines, has been evaluated as a single agent or in combination with phenoxybenzamine for hemodynamic stability with mixed results but not in pregnant patients (27). Currently, it is reserved as monotherapy or in combination with phenoxybenzamine only for cases where all other medication has failed or in cases of large tumours (27).

Invariably, despite extensive experience in the use of antihypertensive medication during pregnancy and the benefits associated with the mother, there is a paucity of evidence with only a few studies suggesting a possible risk of adverse child health outcomes (28). Uncertainty remains around important safety issues for many antihypertensive drugs in pregnancy, despite extensive documentation of use (29).

Importantly, the main challenge in managing hypertension in pregnancy remains the preservation of sufficient uteroplacental circulation, which is autoregulated and remains susceptible to changes in maternal blood pressure (30).

Finally, some commonly prescribed drugs in the peripartum phase or during a Caesarian section (e.g., metoclopramide, steroids, and sympathomimetics), must be avoided due to their potential of resulting in a sudden tumoural release of catecholamines or histamine inducing a pheochromocytoma crisis (31).

It is important to highlight cases when maternal hypertension is not well-controlled (e.g., in unexpected premature labor or undiagnosed pheochromocytoma) resulting in life-threatening emergencies such as acute hypertensive crisis (catecholamine storms), hypertensive encephalopathy, or cardiac ischemia (32). In these circumstances, a rapidly acting $\alpha$-antagonist (e.g., prazosin), calcium channel blockers (e.g., nifedipine or amlodipine), and/or magnesium sulfate can be given safely during pregnancy. Nitroprusside is controversial due to its potential to cause fetal cyanide toxicity (10). Under the above conditions, some complications may occur significantly raising the mortality rate for the mother and child despite quick, appropriate therapy (33).

\section{Prognosis and follow-up}

Cases in which excision can be undertaken generally exhibit better outcomes than when a medical management approach is adopted, and excision is delayed (7). Excision-related complications arise due to unrecognised additional pheochromocytomas while medical management alone may be associated with intrauterine death and the development of metastatic disease (34).
To date, it is unclear the consequences that long-term hypertensive treatment has on the fetus. Nonetheless, the outcomes following long-term hypertensive treatment tend to be satisfactory with a reported fetal and maternal survival rate as high as $85 \%(7,35)$.

Genetic predisposition plays a major role in the overall progression of pheochromocytomas particularly post-excision.

Long-term monitoring of patients despite complete surgical excision remains obligatory. Following the surgical intervention, 1 to 2 weeks postoperatively, fractionated catecholamines and their metabolites need to be measured. Medical treatment is continued until normal biochemical levels are reached and are weaned slowly especially if $\beta$-blockers are used (due to risk of rebound hypertension if abruptly stopped) considering the patient's symptomatology. Annual biochemical controls remain lifelong. In $20 \%$ of cases, essential hypertension requiring medical treatment persists $(6,26)$. In cases where patients undergo bilateral adrenalectomy, lifelong glucocorticoid and mineralocorticoid replacement therapy must be considered.

\section{Conclusions}

Clinical suspicion and meticulous patient history-taking remain the primary lines of defense against a pathology that scarcely appears during pregnancy but remains one with the highest mortality and morbidity rates for both the mother and fetus.

For the positive diagnosis of pheochromocytoma, two elements are required: proof of catecholamine excess and localisation of the tumour(s) while genetic analysis of possible mutations provides additional information and guidance on the potential progress and best therapeutic attitude towards pheochromocytoma.

In terms of biochemical testing, sensitive plasma metanephrine measurements are to be reserved for high a priori risk patients (including those with suspected familial endocrine syndromes), while 24-h urinary total catecholamines and metanephrines can be used for low a priori risk patients. Attention must be drawn to the increased false-positive rate in biochemical testing due to interfering drugs or the falsenegative rate due to dopamine-secreting tumours. In terms of imaging, MRI remains the method of choice throughout pregnancy, without excluding the use of ultrasound during the first trimester.

The extremely low incidence of symptomatic pheochromocytomas in pregnancy as seen by the limited number of published cases, in conjunction with a publication and a selection bias, does not provide a whole inclusive algorithm in addressing them, and we have strived to provide some guidance in suspecting and managing such cases. Regardless, key factors to a successful outcome for both the mother and the newborn include the prompt diagnosis of the pheochromocytoma and complete localisation antenatally, the avoidance of hypertensive crises while maintaining adequate uteroplacental circulation, and the close monitoring and control of the patients' blood pressure. An experienced and multidisciplinary team is required to effectively manage the condition.

In the future, clinical trials are needed to compare the treatment of hypertension in pregnancy with and without 
different classes of antihypertensive drugs. In these evaluations, both the mothers' and the fetuses'/newborns' safety need to be considered in the short and long term. Ultimately, blood-pressure control of the mother and the well-being of the fetus are the determining factors in deciding the time of delivery, with a Caesarean section being the most preferred route. Despite the ever-improving positive outcome rates, pregnancy-associated pheochromocytoma remains a pathology with high mortality and morbidity rates for both the mother and the fetus, especially in the context of hypertensive crises, thus warranting heightened awareness and careful management on behalf of the treating physicians.

\section{Acknowledgements}

Not applicable.

\section{Funding}

No funding was received.

\section{Availability of data and materials}

All data and information were collected through open or paid databases containing published journals.

\section{Authors' contributions}

IP, NZ, CGF, DN, DMT, CAI, APS, AP and MD were equal contributors in collecting the available literature information. IP and NZ composed the final manuscript. IP in addition coordinated the entire effort. All authors read and approved the final manuscript for publication.

\section{Ethics approval and consent to participate}

Not applicable.

\section{Patient consent for publication}

Not applicable.

\section{Competing interests}

The authors declare that they have no competing interests.

\section{Authors' information}

Author ORCID numbers: Irina Pacu: 0000-0002-4253-6810; Nikolaos Zygouropoulos: 0000-0002-8404-6158; Cristian George Furau: 0000-0001-7158-2579; Delia Mirela Tit: 0000-0002-0296-6592; Cringu A. Ionescu: 0000-00034533-6766; Anca Pantea Stoian: 0000-0003-0555-526X; Aida Petca: 0000-0002-4890-0978; Mihai Dimitriu: 0000-00027454-7389.

\section{References}

1. Cunningham FG, Leveno KJ, Bloom SL, Dasche JS, Hoffman BL, Casey BM and Spong CY: Williams Obstetrics. 25th edition. McGraw Hill Education, New York, NY, 2018.
2. Lenders JWM, Eisenhofer G, Mannelli M and Pacak K: Phaeochromocytoma. Lancet 366: 665-675, 2005.

3. Quartermaine G, Lambert K, Rees K, Seed PT, Dhanjal MK, Knight M, McCance DR and Williamson C: Hormone-secreting adrenal tumours cause severe hypertension and high rates of poor pregnancy outcome; a UK obstetric surveillance system study with case control comparisons. BJOG 125: 719-727, 2018.

4. Favier J, Amar L and Gimenez-Roqueplo AP: Paraganglioma and phaeochromocytoma: From genetics to personalized medicine. Nat Rev Endocrinol 11: 101-111, 2015.

5. Fishbein L, Leshchiner I, Walter V, Danilova L, Robertson AG, Johnson AR, Lichtenberg TM, Murray BA, Ghayee HK, Else T, et al: comprehensive molecular characterization of pheochromocytoma and paraganglioma. Cancer Cell 31: 181-193, 2017.

6. Tsirlin A, Oo Y, Sharma R, Kansara A, Gliwa A and Banerji MA: Pheochromocytoma: A review. Maturitas 77: 229-238, 2014.

7. Biggar MA and Lennard TWJ: Systematic review of phaeochromocytoma in pregnancy. Br J Surg 100: 182-190, 2013.

8. Lenders JWM, Langton K, Langenhuijsen JF and Eisenhofer G: Pheochromocytoma and pregnancy. Endocrinol Metab Clin North Am 48: 605-617, 2019.

9. Manger WM: An overview of pheochromocytoma: History, current concepts, vagaries, and diagnostic challenges. Ann N Y Acad Sci 1073: 1-20, 2006.

10. Prete A, Paragliola RM, Salvatori R and Corsello SM: Management of catecholamine-secreting tumors in pregnancy: A review. Endocr Pract 22: 357-370, 2016.

11. Lenders JWM, Pacak K, Walther MM, Linehan WM, Mannelli M, Friberg P, Keiser HR, Goldstein DS and Eisenhofer G: Biochemical diagnosis of pheochromocytoma: Which test is best? JAMA 287: 1427-1434, 2002.

12. Sawka AM, Jaeschke R, Singh RJ and Young WF Jr: A comparison of biochemical tests for pheochromocytoma: Measurement of fractionated plasma metanephrines compared with the combination of 24-hour urinary metanephrines and catecholamines. J Clin Endocrinol Metab 88: 553-558, 2003.

13. Eisenhofer G, Goldstein DS, Walther MM, Friberg P, Lenders JWM, Keiser HR and Pacak K: Biochemical diagnosis of pheochromocytoma: How to distinguish true-from false-positive test results. J Clin Endocrinol Metab 88: 2656-2666, 2003.

14. Sarathi V, Lila AR, Bandgar TR, Menon PS and Shah NS: Pheochromocytoma and pregnancy: A rare but dangerous combination. Endocr Pract 16: 300-309, 2010.

15. Lenders JWM, Duh QY, Eisenhofer G, Gimenez-Roqueplo AP, Grebe SK, Murad MH, Naruse M, Pacak K and Young WF Jr; Endocrine Society: Pheochromocytoma and paraganglioma: An endocrine society clinical practice guideline. J Clin Endocrinol Metab 99: 1915-1942, 2014.

16. Fassnacht M, Arlt W, Bancos I, Dralle H, Newell-Price J, Sahdev A, Tabarin A, Terzolo M, Tsagarakis S and Dekkers OM: Management of adrenal incidentalomas: European society of endocrinology clinical practice guideline in collaboration with the European network for the study of adrenal tumors. Eur J Endocrinol 175: G1-G34, 2016.

17. Young WF Jr and Maddox DE: Spells: In search of a cause. Mayo Clin Proc 70: 757-765, 1995.

18. Pearl J, Price R, Richardson W and Fanelli R; Society of American Gastrointestinal Endoscopic Surgeons: Guidelines for diagnosis, treatment, and use of laparoscopy for surgical problems during pregnancy. Surg Endosc 25: 3479-3492, 2011.

19. Zeiger MA, Thompson GB, Duh QY, Hamrahian AH, Angelos P, Elaraj D, Fishman E and Kharlip J; American Association of Clinical Endocrinologists; American Association of Endocrine Surgeons: The American association of clinical endocrinologists and American Association of endocrine surgeons medical guidelines for the management of adrenal incidentalomas. Endocr Pract 15 (Suppl 1): S1-S20, 2009.

20. Tauzin-Fin P, Sesay M, Gosse P and Ballanger P: Effects of perioperative alpha1 block on haemodynamic control during laparoscopic surgery for phaeochromocytoma. Br J Anaesth 92: 512-517, 2004.

21. NICE: Overview: Hypertension in pregnancy: diagnosis and management. Guidance. https://www.nice.org.uk/guidance/ ng133. Accessed June 25, 2019.

22. Santeiro ML, Stromquist C and Wyble L: Phenoxybenzamine placental transfer during the third trimester. Ann Pharmacother 30: 1249-1251, 1996. 
23. Versmissen J, Koch BCP, Roofthooft DWE, Bosch-Dijksman WT, van den Meiracker AH, Hanff LM and Visser W: Doxazosin treatment of phaeochromocytoma during pregnancy: Placental transfer and disposition in breast milk. Br J Clin Pharmacol 82: $568-569,2016$.

24. Schifferdecker B, Kodali D, Hausner E and Aragam J: Adrenergic shock-an overlooked clinical entity? Cardiol Rev 13: 69-72, 2005.

25. Pacak K: Preoperative management of the pheochromocytoma patient. J Clin Endocrinol Metab 92: 4069-4079, 2007.

26. Stoicescu M, Csepento C, Muţiu G and Bungău S: The role of increased plasmatic renin level in the pathogenesis of arterial hypertension in young adults. Rom J Morphol Embryol 52 (Suppl): S419-S423, 2011

27. Steinsapir J, Carr AA, Prisant LM and Bransome ED Jr: Metyrosine and pheochromocytoma. Arch Intern Med 157: 901-906, 1997.

28. Fitton CA, Steiner MFC, Aucott L, Pell JP, Mackay DF, Fleming $\mathrm{M}$ and McLay JS: In-utero exposure to antihypertensive medication and neonatal and child health outcomes: A systematic review. J Hypertens 35: 2123-2137, 2017.

29. Malha L and August P: Safety of antihypertensive medications in pregnancy: Living with uncertainty. J Am Heart Assoc 8: e013495, 2019

30. van der Weerd K, van Noord C, Loeve M, Knapen MFCM, Visser W, de Herder WW, Franssen G, van der Marel CD and Feelders RA: Endocrinology in pregnancy: Pheochromocytoma in pregnancy: Case series and review of literature. Eur J Endocrinol 177: R49-R58, 2017.
31. Kamoun M, Mnif MF, Charfi N, Kacem FH, Naceur BB, Mnif F, Dammak M, Rekik N and Abid M: Adrenal diseases during pregnancy: Pathophysiology, diagnosis and management strategies. Am J Med Sci 347: 64-73, 2014.

32. Riester A, Weismann D, Quinkler M, Lichtenauer UD, Sommerey S, Halbritter R, Penning R, Spitzweg C, Schopohl J, Beuschlein $\mathrm{F}$ and Reincke M: Life-threatening events in patients with pheochromocytoma. Eur J Endocrinol 173: 757-764, 2015.

33. Desai AS, Chutkow WA, Edelman E, Economy KE and Dec GW Jr: Clinical problem-solving. A crisis in late pregnancy. N Engl J Med 361: 2271-2277, 2009.

34. Snabboon T, Plengpanich W, Houngngam N, Buranasupkajorn P, Plengvidhya N, Sereepapong W, Sunthornyothin S and Shotelersuk V: Concurrent bilateral pheochromocytoma and thoracic paraganglioma during pregnancy. Endocrine 37: 261-264, 2010.

35. Turcan N, Bohiltea RE, Ionita-Radu F, Furtunescu F, Navolan D, Berceanu C, Nemescu D and Cirstoiu MM: Unfavorable influence of prematurity on the neonatal prognostic of small for gestational age fetuses. Exp Ther Med 20: 2415-2422, 2020. 